\title{
Darwin's finches - an adaptive radiation constructed from ancestral genetic modules
}

Carl-Johan Rubin ${ }^{1, *}$, Erik D. Enbody ${ }^{1, *}$, Mariya P. Dobreva ${ }^{2}$, Arhat Abzhanov², Brian W.

Davis $^{3}$, Sangeet Lamichhaney ${ }^{4}$, Mats Pettersson ${ }^{1}$, C. Grace Sprehn ${ }^{1}$, Carlos A. Valle ${ }^{5}$, Karla

Vasco $^{5}$, Ola Wallerman ${ }^{1}$, B. Rosemary Grant ${ }^{6}$, Peter R. Grant ${ }^{6}$ and Leif Andersson ${ }^{1,3,7^{* *}}$

${ }^{1}$ Department of Medical Biochemistry and Microbiology, Uppsala University, Uppsala, Sweden. ${ }^{2}$ Department of Life Sciences, Imperial College London, Silwood Park Campus, SL5 7PY Ascot, UK. ${ }^{3}$ Department of Veterinary Integrative Biosciences, Texas A\&M University, College Station, USA. ${ }^{4}$ Department of Biological Sciences, Kent State University, Kent, OH, USA. ${ }^{5}$ Colegio de Ciencias Biológicas y Ambientales, Galápagos Science Center GSC, Universidad San Francisco de Quito USFQ, Quito, Ecuador. ${ }^{6}$ Department of Ecology and Evolutionary Biology, Princeton University, Princeton, NJ, USA. ${ }^{7}$ Department of Animal Breeding and Genetics, Swedish University of Agricultural Sciences, Uppsala, Sweden.

* These authors contributed equally

**Corresponding author. Email: leif.andersson@imbim.uu.se 


\begin{abstract}
Recent adaptive radiations are models for investigating mechanisms contributing to the evolution of biodiversity. An unresolved question is the relative importance of new mutations, ancestral variants, and introgressive hybridization for phenotypic evolution and speciation. Here we address this issue using Darwin's finches, which vary in size from an $8 \mathrm{~g}$ warbler finch with a pointed beak to a $40 \mathrm{~g}$ large ground finch with a massive blunt beak. We present a highly contiguous genome assembly for one of the species and investigate the genomic architecture underlying phenotypic diversity in the entire radiation. Admixture mapping for beak and body size in the small, medium and large ground finches revealed 28 loci showing strong genetic differentiation. These loci represent ancestral haplotype blocks with origins as old as the Darwin's finch phylogeny (1-2 million years). Genes expressed in the developing beak are overrepresented in these genomic regions. Frequencies of allelic va riants at the 28 loci covary with phenotypic similarities in body and beak size across the Darwin's finch phylogeny. These ancestral haplotypes constitute genetic modules for selection, and act as key determinants of the exceptional phenotypic diversity of Darwin's finches. Such ancestral haplotype blocks can be critical for how species adapt to environmental variability and change.
\end{abstract}

Identification of the factors that promote or constrain the process of adaptive radiation, or the proliferation of forms from a single common ancestor, provide opportunities for understanding the origins of biodiversity. Species that radiate rapidly are thought to share some common features (1), promoting their ability to evolve into diverse forms $(2,3)$, whereas depauperate clades may lack them $(4,5)$. One of these features, evolvability, may be determined in part by the modularity of phenotypic traits (6), allowing some species to exploit ecological opportunity more readily (3). Two factors that influence why some species exhibit greater evolvability than others are phenotypic plasticity and the genetic potential for diversification (3). While the rapid speciation in adaptive radiations provides limited time to generate de novo genetic variation, ancestral polymorphisms can facilitate rapid accumulation of diverse combinations of alleles (7-12). Under this model, ancestral variation is sorted in unique combinations in descendent lineages (13) and/or is transmitted across lineages through introgression $(9,11)$. Hybridization may lead to loss of genetic differentiation $(14$, 15), but may also enhance the potential for selection by increasing phenotypic and genetic variation $(16,17)$. The identification of genetic variants underlying phenotypic variation is essential for understanding the role of ancestral genetic variation in evolutionary change. This 
remains an outstanding challenge when comparing species in the absence of genetic data because causal variants are greatly outnumbered by neutral variants. However, recent adaptive radiations, in particular those that still hybridize, are excellent groups for studying the origins of genetic variation and their effect on phenotype because gene flow has homogenized the genetic background, thus facilitating the identification of loci contributing to phenotypic differences among species $(18,19)$.

The Darwin's finch radiation comprises 18 species, 17 present in Galápagos and one on Cocos Island. The group is highly unusual in that no species is known to have become extinct as a result of human activities, in contrast to some other avian radiations (20). The species have experienced current and historical gene flow (21-24) and diversification involved a key ecological trait, beak morphology, that mediates efficient use of different food sources (insects, seeds of various sizes, cactus fruits, and even blood from other birds) (25). Previous genetic studies have revealed a few loci where ancestral alleles explain variation in beak morphology: $A L X 1$ affecting beak shape (21), a genomic region controlling beak size including $H M G A 2$ and three other genes $(M S R B 3, L E M D 3, W I F 1)(26,27)$, and in addition a number of suggestive loci under selection $(21,26-28)$. Whether other loci mediating phenotypic evolution in this group also represent ancestral variation and their role in phenotypic evolution in the radiation is unknown. Here, we present a high-quality chromosome-scale reference genome and leverage a natural scaling transformation in beak size (29) across three species of ground finches (Geospiza) to identify 28 loci under selection. We show that these haplotype blocks linked to phenotypic divergence are as old as the Darwin's finch phylogeny. These genetic modules have been reused over the last million years, were exchanged by gene flow, and contributed to the rapid phenotypic evolution and speciation among Darwin's finches.

\section{High quality assembly of the Camarhynchus parvulus genome}

The previously reported genome assembly based on Illumina short reads of a medium ground finch (G. fortis) is highly fragmented (30). We therefore decided to develop a high quality, highly contiguous assembly for Darwin's finches by combining long-read data with chromatin contact (HiC) data (Fig. S1). Because of the uncertainty to export tissue samples with intact long DNA molecules from the Galápagos National Park we carried out Oxford Nanopore Technologies (ONT) sequencing at the Galápagos Science Center. Genomic DNA prepared from a male small tree finch (C. parvulus) was of high molecular weight and this individual was selected for Oxford Nanopore Technologies (ONT) sequencing. The close 
evolutionary relationship among all species of Darwin's finches (pairwise interspecies $d_{X Y}$ in the range $0.2-0.3 \%(31))$ implies that this reference assembly can be used across the phylogeny. We generated 35X ONT long read sequence coverage from the reference individual (Fig. S1). We corrected erroneous base calls using linked-read data and generated chromosome-size scaffolds using $\mathrm{HiC}$ data. The resulting assembly is of similar quality to current state-of-the art genome assemblies in contiguity and accuracy (96\% of the sequence assigned to chromosomes, $\mathrm{N} 50=71.1 \mathrm{Mb}, \mathrm{BUSCO}=96.1 \%$ complete, gaps $=0.01 \%$ ) and shows a high degree of conserved synteny to the zebra finch genome assembly (Fig. 1a). Field-collected tissue samples were used to generate RNA-seq data for annotation (Table S1;

Fig. S1). Genome annotation of these data using the Ensembl annotation pipeline (32) generated 17,167 gene modules that include non-coding RNA and microRNA. We further used 25 C. parvulus individuals to generate a linkage-disequilibrium based recombination map (33) (Fig. S2). Consistent with other avian species $(34,35)$, recombination rates are generally elevated at the ends of chromosomes and correlated with nucleotide diversity $\left(R^{2}=\right.$ $0.19, P<0.001)$, particularly on chromosome $\mathrm{Z}\left(R^{2}=0.27, P<0.001\right)$. This relationship is consistent with the widespread effects of background selection known in birds $(35,36)$.

\section{Twenty-eight trait loci explaining phenotypic differentiation}

We used admixture mapping (37) to search for loci contributing to genetic differentiation between three closely related species that differ primarily in a scaling factor for beak and body size from small to large (29): the small, medium and large ground finches ( $G$. fuliginosa, G. fortis and G. magnirostris, respectively) (Fig. S3). This trio was selected based on their striking phenotypic differentiation in beak and body traits alone and low genomewide genetic differentiation (pairwise $F_{S T}=0.02-0.10$ ), because this reduces the background noise due to genetic drift. In this study, we generated whole genome, short read sequence data from 28 individuals from these three species and combined these with previously published samples for a total of 75 birds on 9 islands (mean coverage $=17 \pm 9$, Table S2; Data S1) and applied phenotypic scores of $0,1,2$ to reflect the increasing beak and body size of $G$.

fuliginosa $<$ G. fortis $<$ G. magnirostris, because phenotypic data were not available for each individual. The experimental setup is similar to an earlier study using these three species, but this studied used only one island and reduced representation sequencing (27). Admixture mapping (37) revealed 28 loci that exceeded the significance threshold set by permutation (Fig. 1b) and represent independent loci (Data S2). The size of these regions ranges from thousands of $\mathrm{kb}$ to $2.7 \mathrm{Mb}$ (Fig. 2) and contained between 0 and 35 genes (Data S2). Outlier 
loci were clustered on macrochromosomes and included the previously described $A L X 1$ and $H M G A 2$ loci affecting beak morphology, both located on chromosome $1 \mathrm{~A}$ and only $\sim 7 \mathrm{Mb}$ apart (locus 4 and 9 in Fig. 1b). These loci are separated by a recombination hot-spot (Fig. S2), consistent with previous results that these loci do not show strong linkage disequilibrium $(21,26)$.

Regions of association identified with admixture mapping largely mirrored the results of $F_{S T}$-based contrasts (Fig. 1d) and strongly correlated with per-window estimates in the two contrasts involving G. fuliginosa $\left(R^{2}\right.$ fortis-fuliginosa $=0.84, R^{2}$ magnirostris-fuliginosa $=0.69$, Data S2).

We do not expect a perfect match between the results of admixture mapping and $F_{S T}$, analysis because the former is based on a linear comparison of the trio while the latter is derived from pairwise comparisons between species. In the 28 regions of association, G. fuliginosa and $G$. magnirostris were often homozygous for different haplotypes while G. fortis exhibited intermediate allele frequencies (Fig. S4; Data S3). This is highlighted at the HMGA2 locus on chromosome 1A, where measures of Tajima's D are strongly negative for G. fuliginosa and G. magnirostris, but strongly positive for G. fortis (Fig. S4), consistent with balancing selection maintaining haplotype diversity in the phenotypically variable G. fortis population(25). Nucleotide diversity was reduced in G. magnirostris relative to the genomic background in 23 of the 28 regions (Fig. 1c), consistent with selective sweeps in $G$. magnirostris or an ancestor. These regions also fell in genome-wide low-recombination regions (mean $\rho$ in peaks $=1.4$ compared with mean $\rho$ outside $=2.0$ ), with the exception of one peak on chromosome $25(\rho=4.7)$, consistent with previous studies in Darwin's finches that regions of elevated differentiation often lie in recombination cold spots (31). Low recombination in these regions has likely facilitated the persistence of large haplotype blocks despite high gene flow amongst Darwin's finch species, as predicted from theoretical studies (36).

To explore the extent to which the loci detected in the ground finch contrast also shape phenotypic diversity among tree finches (Camarhynchus), we next performed a similar admixture analysis comparing small, medium and large tree finches (C. parvulus, C. pauper and C. psittacula, respectively), also classified as $0,1,2$, respectively, based on beak and body size. These samples were previously sequenced and include 46 individuals $(n=10-25$ each) from 8 islands. This replicated a signal for the $H M G A 2$ locus affecting beak size $\left(P=4 \times 10^{-16}\right)$ (Fig. S5), as expected from previous work (26). No other locus showed such a striking signal of genetic differentiation, but we noted an overlap of higher genetic differentiation, approaching genome-wide significance, for several of the loci detected in the ground finch 
contrast (Fig. S5). Nevertheless, the identification of regions associated with phenotypic variation in size among Camarhynchus may be hampered by our comparatively smaller sample size than in Geospiza ( $\mathrm{n}=75$ vs. $\mathrm{n}=46$ ).

In order to determine the evolutionary origin of haplotypes at the 28 loci detected in the ground finch contrast, we compared allele frequencies at the most differentiated SNPs across all 18 species of Darwin's finches, two outgroups L. noctis and T. bicolor. We also included the Big Bird hybrid lineage, which was formed by the mating of a G. conirostris male and two G. fortis females(24). These included genome sequences from previously published data and 62 new individuals ( $n=321$ in total, Data S1). If genetic differentiation among Geospiza is caused by de novo mutations that occurred after the split from Camarhynchus, we would expect to find little shared haplotype structure in non-Geospiza species as a consequence of random accumulation of variants. In sharp contrast, the allele frequency comparison revealed a non-random pattern for the most differentiated SNPs at the 28 loci (Fig. 2). Notably, a few G. magnirostris haplotypes are present at a relatively high frequency across the radiation (e.g., 10, 25), while most are consistently highly differentiated from haplotypes in other species except those with relatively large beaks. Furthermore, a large portion of G. magnirostris major alleles, $67 \%(1,273 / 1,914)$, were derived relative to outgroups L. noctis and T. bicolor consistent with selective sweeps in the G. magnirostris lineage (Data S4). Together, these results imply that the "large" and "small" haplotype blocks identified by admixture mapping in Geospiza predates the separation of Geospiza and Camarhynchus. Heterogenous combinations of these haplotypes in two species with large beaks, G. propinqua and the Big Bird lineage (Fig. 2), raise the intriguing possibility that unique combinations of alleles are formed during the speciation process by incomplete lineage sorting (ILS) and/or introgression, and retained by natural selection.

We evaluated the hypothesis that these haplotype blocks are old by first generating a neighbor-joining genetic distance tree for the entire radiation by concatenating the most differentiated SNPs from each genomic region. The topology of this tree matches previous species-tree reconstructions using other phylogenetic models $(21,24)$. We predicted that, if these haplotypes carry causative mutations for species phenotypes, then phenotypically similar species will cluster together in this "haplotype tree". We compared the topology of this tree with a species tree based on 4.9 million high-quality SNPs from the rest of the genome and found them to be discordant (Fig. 3a); individual trees for each of the 28 loci are given in Fig. S6. We further analysed whether allele frequencies of differentiated SNPs at each of the 28 loci were more concordant with the haplotype tree or with the species tree and 
concluded that, for all loci except two, allele frequencies were more concordant with the haplotype tree (Fig. S7). In the species tree, the earliest divergence involves the warbler finches and all other finches in the radiation (Fig. 3a). In contrast, in the haplotype tree, the most divergent grouping separates the largest ground finches (G. magnirostris, G. fortis and G. conirostris) from all other species. Further, G. fuliginosa and G. acutirostris cluster with Pinaroloxias inornata (the Cocos Island finch), which all share a common small phenotype (small bodies and small pointed beaks), but are not monophyletic on the species tree. At all 28 loci, except one (\#10), the haplotypes present in P. inornata are more similar to those in G. fuliginosa than in G. magnirostris (Fig. 2). This implies that the finch that colonized Cocos Island carried the small variant alleles at most of these 28 loci and subsequently diverged through local adaptation and genetic drift in geographical isolation from other ancestral finch populations. In contrast, the small and medium ground finches, two sympatric species that differ in feeding ecologies and exchange genes (25), are essentially indistinguishable genetically, except at the 28 loci under selection (Fig. S3b). Finally, we confirmed deep divergence (mean age $1.3 \pm 0.5 \mathrm{MYA}$ ) of the major haplotypes in $G$. fuliginosa and G. magnirostris by estimating the time to the most recent common ancestor at each locus using the net frequency of nucleotide substitutions $\left(d_{a}\right)$ and the estimated substitution rates in Darwin's finches (21) (Fig. 3b).

\section{Introgression of ancestral haplotypes}

The presence of distinct combinations of haplotypes across the phylogeny indicates ILS or introgression. The Genovesa cactus finch G. propinqua has the fourth largest beak of all Geospiza, but the pointed beak characteristic of other cactus finches, and carries a mix of large and small haplotypes (Fig. 2). Gene flow from G. magnirostris to G. propinqua has been implicated from field observations (10). We estimated the fraction of introgression from G. magnirostris to G. propinqua on Genovesa using $d_{f}$, which incorporates $d_{X Y}$ into an extension of ABBA/BABA D statistics (38), and found that regions of high G. magnirostris similarity share an excess of derived alleles (Fig. 3c), and often reduced genetic divergence $d_{X Y}$ (Data S5), consistent with introgression. The role of gene flow in generating distinct combinations of haplotypes is also evident in the Big Bird lineage (Fig. 2). The Big Bird lineage is characterized by an unusually large beak on a relatively small body (24). The combination of $G$. conirostris, a sister species to G. magnirostris, and G. fortis alleles resulted in a unique phenotype and genotype, with most loci sharing greater affinities with $G$. conirostris than with G. fortis (Fig. 2). It remains an outstanding challenge to distinguish 
between ILS and introgression using D statistics (39), but our results and field observations strongly imply that gene flow can transfer these haplotypes among species, as previously demonstrated for $A L X 1$ (22).

\section{Trait loci are enriched for developmental genes}

Ancient haplotypes may be retained in descendent species even if they are neutral. However, the loci identified here segregate with phenotypic variation among the species and are expected to contain alleles important for beak and body size variation. We conducted an enrichment analysis using mouse orthologs for the genes in the vicinity of the 28 loci using the software GREAT (40) and found that deleterious mutations at these loci were significantly associated with abnormal development of cartilage and bones (Fig. 4a; Fig. S8). Furthermore, these mouse orthologs were significantly enriched for genes expressed during craniofacial and limb development, which is consistent with our expectation that genetic changes at many of these 28 loci affect beak development. The enrichment includes genes spread across the 28 loci (Data $\mathbf{S 2}$ ).

Because of the difficulties in interpreting gene-by-term enrichment data (41), we performed two types of analyses to validate gene expression. RNA-seq of upper beaks from 9 embryonic day 7 (E7) Darwin's finch embryos representing 6 species confirmed that a number of the genes within the 28 loci are expressed in the developing beak and these loci were 14-fold enriched for genes with higher expression levels $(M \geq 5=$ fold change $\geq 32)$ in the developing beak compared with other tissues $\left(\chi^{2}\right.$-test, $P=7.4 \times 10^{-24}$, d.f. $=1$; Fig. $\left.4 \mathbf{b}\right)$. We carried out in situ hybridization (ISH) of two candidates for craniofacial development ( $A L X 1$ locus 7 and RUNX2-locus 24). ISH data on a total of 7 zebra finch (Taeniopygia gutatta) and 27 Darwin's finch embryos (of 9 species) revealed that $A L X 1$ expression was strongly biased to the beak region over the developmental period when beak size and shape are established (E6-E7)(42), Fig. 4c; Figs S9, S10a). Additionally, we confirmed a similar expression pattern in zebra finch embryos for $R U N X 2$ (alias $O S F 2$ ) - an essential gene for ossification of the mesenchyme (43) (Fig. S10b) located within a strong signal of differentiation among Geospiza (Fig. S4b).

\section{Discussion}

We have identified ancestral haplotypes at 28 loci that have evolved by natural selection, shaping phenotypic diversity among Darwin's finches throughout their evolution. Our functional characterization of these loci contributes to a growing body of literature suggesting 
that genetic differences between species of Darwin's finches are enriched for genes involved in the key pathways for growth and beak development $(21,26-28,42)$. Together these results extend the key role for the beak of the finch in ecological adaptation in this group. In this study, we show that the distribution of these genetic modules across the phylogeny reflects natural selection and most likely both incomplete lineage sorting and introgression $(21,22)$. Importantly, it is not only the presence/absence of these haplotype blocks that affects the phenotype but their frequency within species, illustrated by G. fortis that have intermediate haplotype frequencies at many of these loci (Fig. 2; Data S4). Intermediate frequencies, indicative of balanced polymorphism, provide the underlying variation for selection to sort adaptive haplotypes during speciation. Our findings support previous suggestions that ancestral variants contribute to phenotypic diversity, as indicated for pigmentation phenotypes among other songbird species $(8,13)$, colour morphs in the common wall lizard (44), colour patterns in Heliconius butterflies (11), various phenotypic traits in cichlids $(7,9)$, craniofacial morphology in pupfish (45), winter coat in snow-shoe hares (46), and adaptation to high altitude in humans (47). That ancestral variation can be retained in large populations preceding speciation is illustrated in Atlantic herring and stickleback where ecotypes show differences in the frequency of haplotype blocks at hundreds of loci, all underlying ecological adaption $(48,49)$.

Characteristic features of these 28 loci are the large size of the haplotype blocks, often spanning hundreds of kilobases, and their ancient origins, 1-2 million years ago (Fig. 3b). This is in contrast to another ancestral polymorphism in Darwin's finches, at the BCO2 locus controlling nestling beak colour, where a single base change constitutes the likely causal mutation in the absence of haplotype structure (50). The identification of causal variants within the haplotype blocks described here is challenging because of strong linkage disequilibrium among many sequence variants within each region. Such large haplotypes could include structural variants, which have been proposed as a key determinant of adaptive evolution and speciation $(51,52)$, but these 28 loci do not represent large inversions $(>5 \mathrm{Mb})$ and tended to be relatively small (0.1-2.7 Mb) compared to e.g., supergenes $(51,53)$. Furthermore, none of the loci exhibit the sharp borders in our association analysis that is characteristic of inversions maintained as balanced polymorphisms $(48,53)$.

The block structures at the 28 loci most likely reflect large-effect haplotypes composed of clusters of multiple causal variants that have accumulated during the evolution of Darwin's finches (54), similar to the evolution of alleles in domestic animals by the sequential accumulation of causal mutations during the last 10,000 years (55). The 
occurrence of these haplotype blocks in low recombination regions likely facilitated their evolution (Data S2). The reuse of ancestral genetic modules is a much faster route to adaptive change than the slow accumulation of adaptive de novo mutations (7). Our study is comprehensive in surveying genomic variation across all 18 extant species of a single adaptive radiation, and yet the principle finding, of repeated reassembly of ancient haplotype blocks in the formation of species, is likely to be a general feature of rapid radiations $(8,45)$ and of general importance for how species adapt to environmental variability and change (25).

\section{Author contributions}

P.R.G. and B.R.G. collected the blood samples. L.A., C-J.R., P.R.G., B.R.G. and E.D.E. conceived the study. C.J.R, S.L. and K.V. performed ONT sequencing on Galápagos. C.J.R. and E.D.E. were responsible for the bioinformatic analysis. A.A. and M.P.D. collected embryonic material, prepared RNA samples and performed ISH. B.W.D. and M.P. contributed to the bioinformatic analysis. C.G.S. and O.W. contributed to experimental work. C.A.V. contributed to sample collection. L.A., E.D.E., B.R.G., P.R.G, and C-J.R. wrote the paper with input from other authors. All authors approved the manuscript before submission.

\section{Data availability statement}

The ONT reads and the Illumina reads have been submitted to the short reads archive (http://www.ncbi.nlm.nih.gov/sra) under BioProject PRJNA743742.

\section{Code availability statement}

The analyses of data have been carried out with publicly available software and all are cited in the Methods section. Code associated with bioinformatic analyses are available at:

\section{https://github.com/erikenbody/Darwins_finch_comparative_genomics.}

\section{Competing interest statement}

The authors declare no competing interest.

\section{Acknowledgements}

We thank Ashley Sendell-Price for helpful discussion on the manuscript and Fan Han for initial bioinformatic analysis. The collection of blood samples, funded by National Science Foundation (NSF), was conducted with annual permits from the Galápagos National Parks 
Directorate, with approval of Princeton University's Animal Care Committee and in accordance with its protocols, and supported logistically by the Charles Darwin Research Station in Galápagos. We thank Oxford Nanopore Technologies for lending us sequencing equipment and for technical assistance related to library preparation and sequencing. Denye Ogeh and Fergal Martin at EMBL-EBI performed gene annotation. Blood samples were collected on San Cristóbal Island under permit number MAE-DNB-CM-2016-0041 to C.A.V., for which we thank Ministerio del Ambiente de Ecuador. Logistical support for researchers to enter the Galápagos and perform laboratory work was provided by GSCUniversidad San Francisco Quito. Tissue samples for expression analysis were collected with permissions PC-08-13 and PC-34-14 from Galápagos National Park; and MAE-DNB-CM2016-0043 from Ministerio del Ambiente de Ecuador. The project was financially supported by Vetenskapsrådet and Knut and Alice Wallenberg Foundation. The National Genomics Infrastructure (NGI)/Uppsala Genome Center provided service in massive parallel sequencing and the computational infrastructure was provided by the Swedish National Infrastructure for Computing (SNIC) at UPPMAX partially funded by the Swedish Research Council through grant agreement no. 2018-05973.

\section{Author Information}

The authors declare no competing financial interests. Correspondence and requests for materials should be addressed to L.A. (leif.andersson@imbim.uu.se).

\section{References and Notes}

1. R. G. Gillespie et al., Comparing adaptive radiations across space, time, and taxa. Journal of Heredity 111, 1-20 (2020)10.1093/jhered/esz064).

2. D. Schluter, The Ecology of Adaptive Radiation. (Oxford University Press, Oxford, U.K., 2000).

3. J. T. Stroud, J. B. Losos, Ecological opportunity and adaptive radiation. Annual Review of Ecology, Evolution, and Systematics 47, 507-532 (2016).

4. I. J. Lovette, E. Bermingham, R. E. Ricklefs, Clade-specific morphological diversification and adaptive radiation in Hawaiian songbirds. Proceedings of the Royal Society of London. Series B: Biological Sciences 269, 37-42 (2002)doi:10.1098/rspb.2001.1789).

5. P. R. Grant, B. R. Grant, How and why species multiply. The radiation of Darwin's finches., (Princeton University Press, Princeton, N.J., USA, 2008).

6. D. Melo, A. Porto, J. M. Cheverud, G. Marroig, Modularity: genes, development and evolution. Annu Rev Ecol Evol Syst 47, 463-486 (2016)10.1146/annurev-ecolsys-121415-032409). 
7. D. A. Marques, J. I. Meier, O. Seehausen, A combinatorial view on speciation and adaptive radiation. Trends Ecol Evol 34, 531-544 (2019); published online EpubJun (10.1016/j.tree.2019.02.008).

8. K. F. Stryjewski, M. D. Sorenson, Mosaic genome evolution in a recent and rapid avian radiation. Nat Ecol Evol 1, 1912-1922 (2017); published online EpubDec (10.1038/s41559-017-0364-7).

9. J. I. Meier et al., Ancient hybridization fuels rapid cichlid fish adaptive radiations. Nat Commun 8, 14363 (2017); published online EpubFeb 10 (10.1038/ncomms14363).

10. R. F. Guerrero, M. W. Hahn, Speciation as a sieve for ancestral polymorphism. Mol Ecol 26, 5362-5368 (2017); published online EpubOct (10.1111/mec.14290).

11. N. B. Edelman et al., Genomic architecture and introgression shape a butterfly radiation. Science 366, 594-599 (2019); published online EpubNov 1 (10.1126/science.aaw2090).

12. R. D. H. Barrett, D. Schluter, Adaptation from standing genetic variation. Trends in Ecology \& Evolution 23, 38-44 (2008); published online Epub2008/01/01/ (https://doi.org/10.1016/j.tree.2007.09.008).

13. S. P. Turbek et al., Rapid speciation via the evolution of pre-mating isolation in the Iberá Seedeater. Science 371, (2021); published online EpubMar 26 (10.1126/science.abc0256).

14. M. Todesco et al., Hybridization and extinction. Evolutionary Applications 9, 892-908 (2016)https://doi.org/10.1111/eva.12367).

15. A. M. Kearns et al., Genomic evidence of speciation reversal in ravens. Nature Communications 9, 906 (2018); published online Epub2018/03/02 (10.1038/s41467-018-03294-w).

16. P. R. Grant, B. R. Grant, Hybridization increases population variation during adaptive radiation. Proc Natl Acad Sci U S A 116, 23216-23224 (2019); published online EpubNov 12 (10.1073/pnas.1913534116).

17. P. R. Grant, B. R. Grant, Phenotypic and genetic effects of hybridization in Darwin's finches. Evolution 48, 297-316 (1994); published online EpubApr (10.1111/j.1558-5646.1994.tb01313.x).

18. N. H. Barton, G. M. Hewitt, Adaptation, speciation and hybrid zones. Nature 341, 497-503 (1989); published online EpubOct 12 (10.1038/341497a0).

19. B. A. Payseur, Using differential introgression in hybrid zones to identify genomic regions involved in speciation. Mol Ecol Resour 10, 806-820 (2010); published online EpubSep (10.1111/j.1755-0998.2010.02883.x).

20. C. Van Riper III, J. M. Scott, S. Conant, Evolution, ecology, and management of Hawaiian birds: a vanishing avifauna. Studies in Avian Biology 22, 1-428 (2001).

21. S. Lamichhaney et al., Evolution of Darwin's finches and their beaks revealed by genome sequencing. Nature 518, 371-375 (2015).

22. S. Lamichhaney et al., Female-biased gene flow between two species of Darwin's finches. Nature Ecology \& Evolution 4, 979-986 (2020); published online Epub2020/07/01 (10.1038/s41559-020-1183-9).

23. H. L. Farrington, L. P. Lawson, C. M. Clark, K. Petren, The evolutionary history of Darwin's finches: speciation, gene flow, and introgression in a fragmented landscape. Evolution 68, 2932-2944 (2014).

24. S. Lamichhaney et al., Rapid hybrid speciation in Darwin's finches. Science 359, 224-228 (2018)10.1126/science.aao4593). 
25. P. R. Grant, B. R. Grant, 40 Years of evolution. Darwin's finches on Daphne Major island., (Princeton University Press, Princeton, N.J., USA, 2014).

26. S. Lamichhaney et al., A beak size locus in Darwin's finches facilitated character displacement during a drought. Science 352, 470-474 (2016).

27. J. A. Chaves et al., Genomic variation at the tips of the adaptive radiation of Darwin's finches. Molecular Ecology 25, 5282-5295 (2016)https://doi.org/10.1111/mec.13743).

28. L. P. Lawson, K. Petren, The adaptive genomic landscape of beak morphology in Darwin's finches. Molecular Ecology 26, 4978-4989 (2017)https://doi.org/10.1111/mec.14166).

29. O. Campàs, R. Mallarino, A. Herrel, A. Abzhanov, M. P. Brenner, Scaling and shear transformations capture beak shape variation in Darwin's finches. Proc Natl Acad Sci USA 107, 3356-3360 (2010)10.1073/pnas.0911575107).

30. G. Zhang, P. Parker, B. Li, H. Li, J. Wang, The genome of Darwin's Finch (Geospiza fortis). GigaScience, (2012)http://dx.doi.org/10.5524/100040).

31. F. Han et al., Gene flow, ancient polymorphism, and ecological adaptation shape the genomic landscape of divergence among Darwin's finches.

Genome Research 27, 1004-1015 (2017); published online EpubJune 1, 2017 (10.1101/gr.212522.116).

32. B. L. Aken et al., The Ensembl gene annotation system. Database (Oxford) 2016, (2016)10.1093/database/baw093).

33. A. H. Chan, P. A. Jenkins, Y. S. Song, Genome-wide fine-scale recombination rate variation in Drosophila melanogaster. PLOS Genetics 8, e1003090 (2012)10.1371/journal.pgen.1003090).

34. M. A. Groenen, H. H. Cheng, N. Bumstead, e. al., A consensus linkage map of the chicken genome. Genome Research 10, 137-147 (2000).

35. S. Singhal et al., Stable recombination hotspots in birds. Science 350, 928932 (2015)10.1126/science.aad0843).

36. B. Charlesworth, M. T. Morgan, D. Charlesworth, The effect of deleterious mutations on neutral molecular variation. Genetics 134, 1289-1303 (1993); published online EpubAug (

37. X. Zhou, M. Stephens, Genome-wide efficient mixed-model analysis for association studies. Nature Genetics 44, 821-824 (2012); published online Epub2012/07/01 (10.1038/ng.2310).

38. B. Pfeifer, D. D. Kapan, Estimates of introgression as a function of pairwise distances. BMC Bioinformatics 20, 207 (2019); published online Epub2019/04/23 (10.1186/s12859-019-2747-z).

39. S. H. Martin, J. W. Davey, C. D. Jiggins, Evaluating the use of ABBA-BABA statistics to locate introgressed loci. Molecular Biology and Evolution 32, 244257 (2014)10.1093/molbev/msu269).

40. C. Y. McLean et al., GREAT improves functional interpretation of cisregulatory regions. Nat Biotech 28, 495-501 (2010)http://www.nature.com/nbt/journal/v28/n5/abs/nbt.1630.html\#suppleme ntary-information).

41. P. Pavlidis, J. D. Jensen, W. Stephan, A. Stamatakis, A critical assessment of storytelling: gene ontology categories and the importance of validating genomic scans. Molecular Biology and Evolution 29, 3237-3248 (2012)10.1093/molbev/mss136). 
42. R. Mallarino et al., Two developmental modules establish 3D beak-shape variation in Darwin's finches. Proc. Natl Acad. Sci. USA 198, 4057-4062 (2011).

43. P. Ducy, R. Zhang, V. Geoffroy, A. L. Ridall, G. Karsenty, Osf2/Cbfa1: a transcriptional activator of osteoblast differentiation. Cell 89, 747-754 (1997); published online EpubMay 30 (10.1016/s0092-8674(00)80257-3).

44. P. Andrade et al., Regulatory changes in pterin and carotenoid genes underlie balanced color polymorphisms in the wall lizard. Proceedings of the National Academy of Sciences 116, 5633-5642 (2019)10.1073/pnas.1820320116).

45. E. J. Richards et al., A vertebrate adaptive radiation is assembled from an ancient and disjunct spatiotemporal landscape. Proc Natl Acad Sci U S A 118, (2021); published online EpubMay 18 (10.1073/pnas.2011811118).

46. M. R. Jones et al., Adaptive introgression underlies polymorphic seasonal camouflage in snowshoe hares. Science 360, 1355-1358 (2018); published online EpubJun 22 (10.1126/science.aar5273).

47. X. Zhang et al., The history and evolution of the Denisovan-EPAS1 haplotype in Tibetans. Proc Natl Acad Sci U S A 118, (2021); published online EpubJun 1 (10.1073/pnas.2020803118).

48. F. Han et al., Ecological adaptation in Atlantic herring is associated with large shifts in allele frequencies at hundreds of loci. eLife 9, e61076 (2020); published online EpubDec 4 (10.7554/eLife.61076).

49. G. A. Roberts Kingman et al., Predicting future from past: The genomic basis of recurrent and rapid stickleback evolution. Science Advances 7, eabg5285 (2021)10.1126/sciadv.abg5285).

50. E. D. Enbody et al., Transspecies beak color polymorphism in the Darwin's finch radiation. bioRxiv, 2021.2001.2019.426595 (2021)10.1101/2021.01.19.426595).

51. M. Todesco et al., Massive haplotypes underlie ecotypic differentiation in sunflowers. Nature 584, 602-607 (2020); published online EpubAug (10.1038/s41586-020-2467-6).

52. D. M. Hooper, T. D. Price, Chromosomal inversion differences correlate with range overlap in passerine birds. Nat Ecol Evol 1, 1526-1534 (2017); published online EpubOct (10.1038/s41559-017-0284-6).

53. S. Lamichhaney, G. Fan, F. Widemo, U. Gunnarsson, D. Schwochow Thalmann, Structural genomic changes underlie alternative reproductive strategies in the ruff (Philomachus pugnax). Nat Gen 48, 84-88 (2016).

54. M. S. Almen et al., Adaptive radiation of Darwin's finches revisited using whole genome sequencing. BioEssays 38, 14-20 (2016).

55. L. Andersson, Molecular consequences of animal breeding. Curr. Opin. Genet. Dev. 23, 295-301 (2013)http://dx.doi.org/10.1016/j.gde.2013.02.014).

56. C. Darwin, Journal of Researches into the Geology and Natural History of the Various Countries visited by H.M.S. Beagle, under the Command of Captain FitzRoy, R.N. from 1832 to 1936. (Henry Colbum, London, 1839).

57. E. Paradis, K. Schliep, ape 5.0: an environment for modern phylogenetics and evolutionary analyses in R. Bioinformatics 35, 526-528 (2019); published online EpubFeb 1 (10.1093/bioinformatics/bty633).

58. L. J. Revell, phytools: an R package for phylogenetic comparative biology (and other things). Methods in Ecology and Evolution 3, 217-223 (2012)https://doi.org/10.1111/j.2041-210X.2011.00169.x). 


\section{Figure captions:}

Fig. 1. Genome assembly and genetic differentiation among three species of Darwin's finches. (a) Illustration of conserved synteny to zebra finch (T. guttata). (b) Genome-wide admixture mapping using three species sorted ascendingly by beak and body size: $0=$ fuliginosa, $1=$ fortis and 2 =magnirostris. The dotted red line indicates the significance threshold set by permutation. Illustrations of the three species are adapted from P.R Grant and Darwin(56). (c) Boxplot showing the difference in nucleotide diversity between the regions of association marked in (b) and regions outside the main area of association. Centreline indicates the median, bounded by the $25^{\text {th }}$ and $75^{\text {th }}$ percentile, with whiskers extending to $1.5 \mathrm{x}$ the interquartile range. (d) Genome-wide $F_{S T}$ for all three possible pairwise combinations of Geospiza considered here. Lines are coloured by the comparison of interest. Many highly divergent regions are shared between contrasts and overlap with regions of association in (b) (Data S2 and S3).

Fig. 2. Haplotype variation across the Darwin's finch phylogeny. The heatmap displays average delta allele frequency (DAF) based on 34-328 SNPs/locus for each species compared to G. magnirostris, the species with the largest beak. On the right, bill size is presented according to a principal component analysis of three beak dimensions averaged across all island populations for each species. Only Geospiza species are shown. Above, the size of each genomic region (in $\mathrm{Mb}$ ) is marked in a dot plot. Right, finch illustrations reproduced by permission of Lynx Edicions.

Fig. 3: Characterization of 28 adaptive loci. (a) Left, a neighbor-joining tree for all species of Darwin's finches based on 4.9 million SNPs. The tree was converted to a chronogram using ape(57) and branching times are reproduced from Lamichhaney et al.(21). Right, a neighborjoining tree for the 28 concatenated loci identified in the association analysis. Species names are coloured by a-priori clade assignments and a co-phylo diagram $(58)$ highlights changes in topology between the trees. (b) Time to most recent common ancestor between G. fuliginosa and $G$. magnirostris haplotypes for all 28 loci. Time was estimated by the conversion of genetic divergence $\left(d_{\mathrm{a}}\right)$ to time using $T=d_{\mathrm{a}} /(2 \mu)$ and a mutation rate of $2.04 \times 10^{-9}(\operatorname{Ref} .(21))$. The horizontal line marks 900,000 years, the approximate time of divergence between warbler finches and all other finches in the radiation(21). (c) Fraction of introgression from $G$. magnirostris to G. propinqua on Genovesa island for each of the 28 loci, as measured by $d_{f}$. Loci are arranged by the strength of the introgression and a line is drawn at the median of genomewide $d_{f}=0.04$. The trio arrangement is written on the graph and ABBA/BABA statistics listed under methods. In (b) and (c) error bars represent $95 \%$ confidence intervals.

Fig. 4. Enrichment analysis and gene expression. (a) Annotation term enrichment analysis. GREAT(40) was used to screen for enrichment of gene annotation terms associated with the 28 differentiated regions. $-\log 10$ Bonferroni corrected $P$-values of significantly enriched terms are shown on the $\mathrm{X}$-axis. Fold enrichment is indicated using dot colours. Dot sizes indicate numbers of genes belonging to each annotation term. (b) Gene expression in Darwin's finch upper beak vs. other tissues. RNAseq gene expression levels in upper beak (n samples $=9$ ) were compared with expression levels in non-craniofacial tissues ( $\mathrm{n}$ samples $=7, \mathrm{n}$ tissues $=4)$ ). $\log 10 P$-values for differential expression are shown on the $\mathrm{y}$-axis and M-values ( $\log 2$ (fold change beak samples vs. other tissues)) are shown on the $\mathrm{x}$-axis. Dotted boxes show genes with M-values $<-5$ and $>+5$, representing genes with lower (left) and higher (right) expression in beak samples (right) 
compared with other tissues. The asymmetry in - $\log 10(P$-values $)$ is a result of comparing one tissue (beak) to several other tissues. Gene names are shown for selected genes. * Separate genes belonging to a gene cluster of scale keratins. ** is a gene of uncertain function (see Data S6). (c) In situ hybridization. Top: schematic representation of the expression pattern of $A L X 1$ (blue) in E7 embryos of zebra finch $(\mathrm{n}=5)$ and Darwin's finches (9 species, $\mathrm{n}=21)$. Bottom: mRNA expression of $A L X 1$ (dark purple) in mid-face longitudinal sections through the heads of Darwin's finch embryos. The developing beak region is shown. Magnified area of the left images is shown on the right. Scale bar: $250 \mu \mathrm{m}$. 
$a$

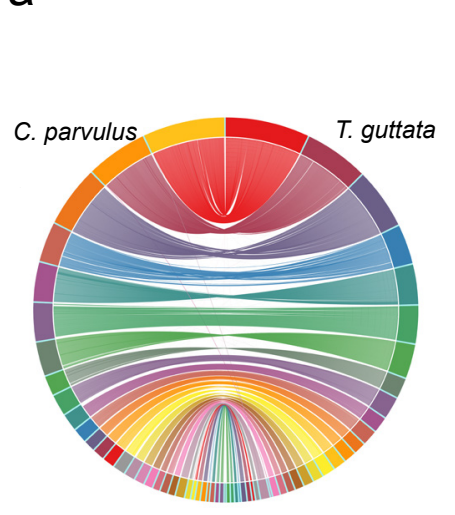

C$$
\text { C }
$$

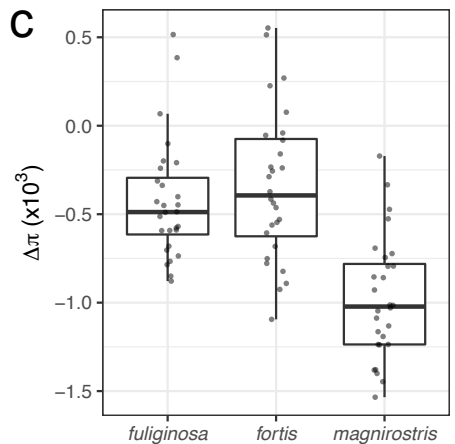

Figure 1 b

Peak \#:

10-17

25

28
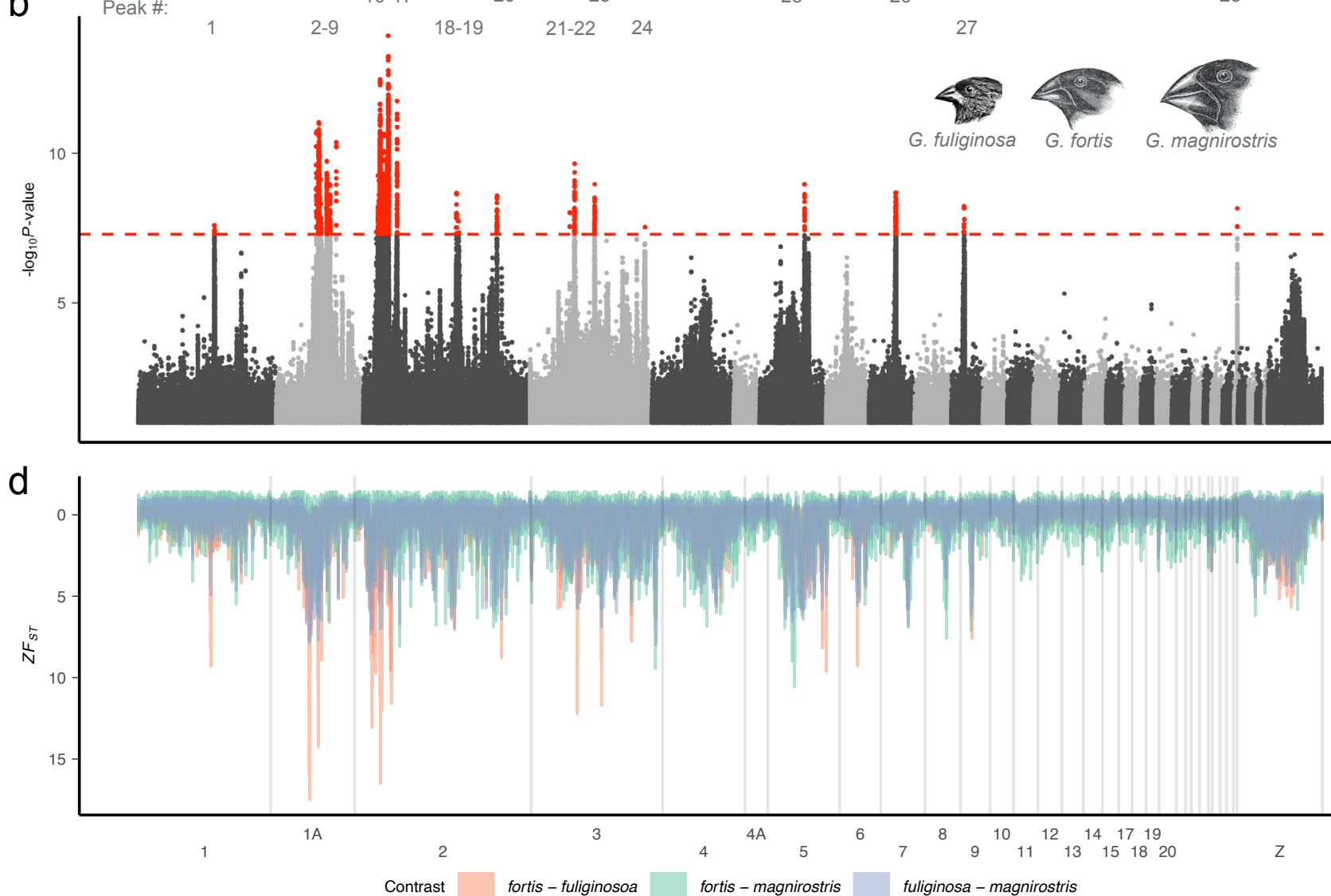
Full-genome"
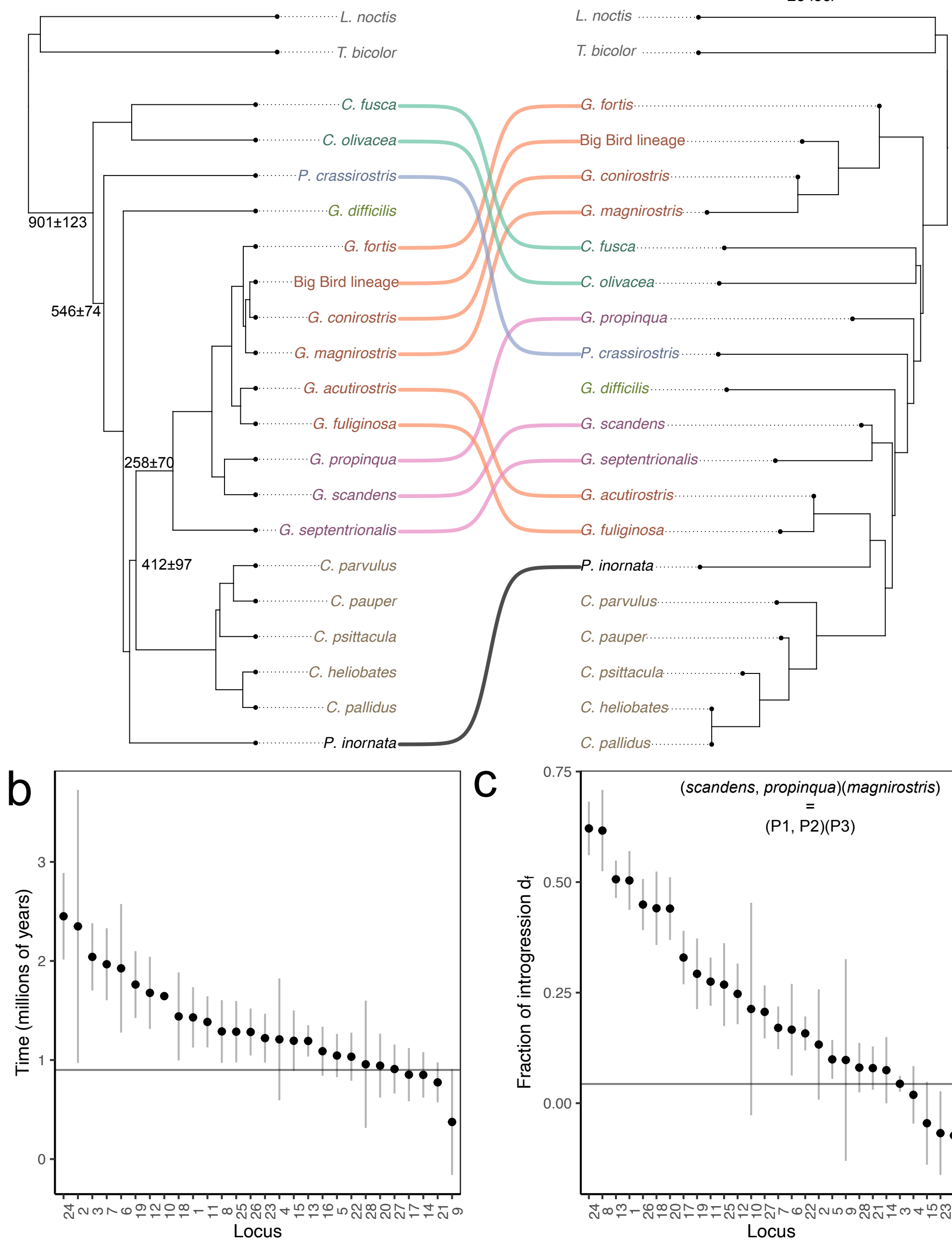

C

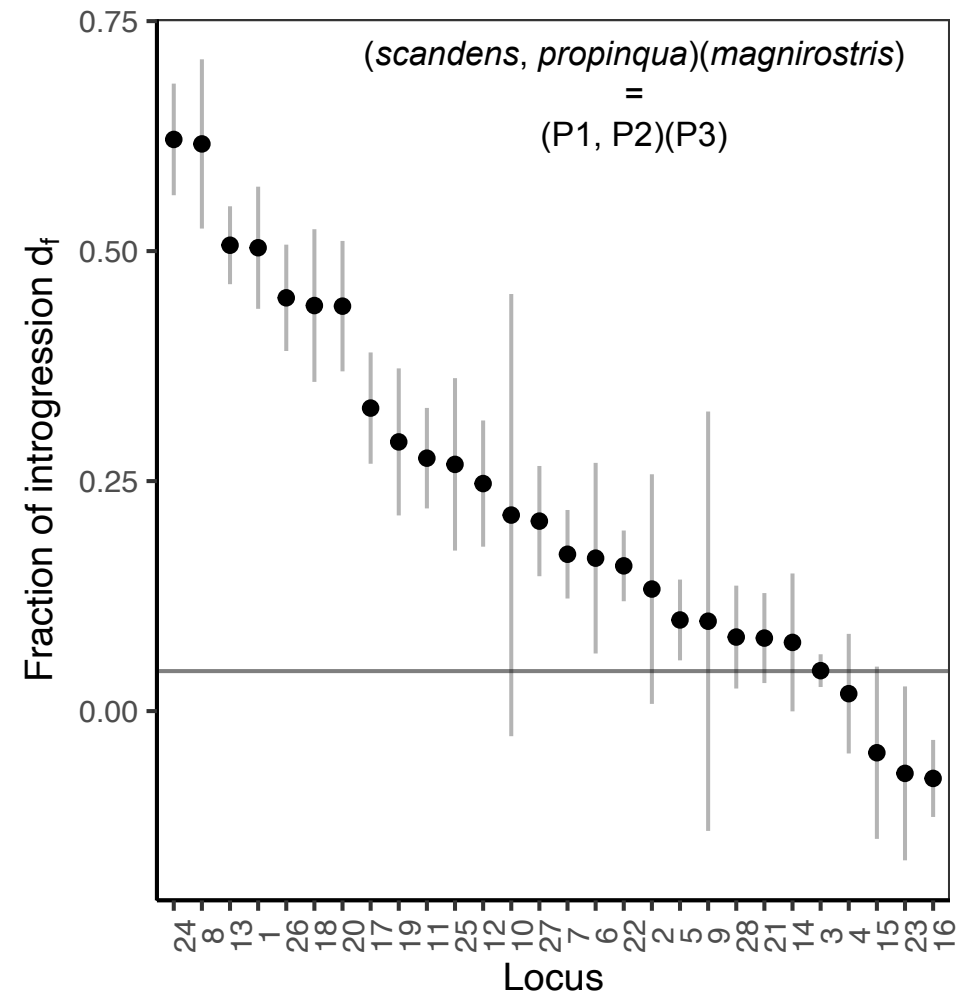

\section{Figure 3}


